\title{
Design of Network Data Transmission Mixed Model Based on Hypothesis Model and Threat Model
}

\author{
Jianying Wang
}

\author{
School of Information Science \& Technology, Zhaotong University, Zhaotong, 65700, China
}

\begin{abstract}
Keywords: Design, Network Data Transmission Mixed Model, Hypothesis Model, Threat Model
\end{abstract}
\begin{abstract}
The data communication network is a system composed of data terminals, data transmission equipment, digital exchange equipment and digital communication lines distributed everywhere. The contents of the communication are binary coded letters, numbers, symbols, and digitized sound and image information. The data communication network and the computer combine to form a computer network, which can use the computer connected to the network and communicate with any other users in the network.
\end{abstract}

\section{Analysis of Data Transmission in Network}

We are using the Internet every day, how is the data on our computer through the Internet to another computer? Write your own understanding, there may be a lot of details cannot be very clear! Hope in the future can make it more perfect! There are wrong places also please correct me.

We know that the TCP / IP protocol used in today's Internet is based on the seven-layer reference model of the OSI (open system interconnection), although not fully compliant, from top to bottom, respectively, for the application layer presentation layer session layer Transport layer network layer Data link layer and physical layer. The data link layer is divided into two sub-layers, namely, logical link control (LLC) and media access control (MAC), which is usually the MAC layer. The nodes in the node are initialized to prevent the connection from being interrupted and maintain reliable communication. The .MAC layer is used to verify the address information contained in each frame. The following will be analyzed. Also understand that the router is at the network layer while the NIC is in the data link layer.

We know that ARP (Address Resolution Protocol) is used as the underlying protocol for IP address to physical address conversion. In the Ethernet, all access to the IP eventually converted to access to the MAC address of the network card. If the host A's ARP list, to the host B's IP address and MAC address corresponds to the incorrect, sent by the A packet to the B packet will be sent to the wrong MAC address, of course, cannot successfully reach B, the result is A and B simply cannot to communicate.

First of all, we analyze the situation in the same network segment. Assuming that two computers are named A and B, respectively, A need to send data to phase B, A host first the target device B's IP address and its own subnet mask "and" operation to determine whether the target device with their own Located in the same network segment. If the target device is in the same network segment and A does not obtain the MAC address information corresponding to the IP address of the target device $\mathrm{B}$, the source device (A) sends ARP in the form of the second layer broadcast (the destination MAC address is all 1) Request packet, which contains the IP address of the source device (A) and the target device (B) in the ARP request packet. All the other devices in the same network segment can receive and analyze the ARP request packet. If the destination IP address of a device discovery packet is the same as its own IP address, it sends an ARP response packet to the source device, the source device obtains the MAC address information of the target device. In order to reduce the broadcast volume, the network device through the ARP table in the cache to save IP and MAC address mapping information. In the process of ARP request and response, both sides of the communication of each other's MAC address and IP address of the correspondence relationship in the respective ARP table to use in the subsequent communication. The ARP table uses the aging mechanism to remove the mapping between IP and MAC addresses that have not been used for a period of time. 
If the middle to go through the switch, according to the principle of the switch, it is directly to the data sent to the corresponding port, then you must keep a database, including all ports connected to the MAC address of the card. It obtains the MAC address of the target B by analyzing the packet header information of the Ethernet packet (which contains the information such as the original MAC address, the destination MAC address, the length of the information, etc.). Then find the address table stored in the switch, the port corresponding to the MAC address ) to confirm that the MAC address of the network card connected to which port, and then send the packet to the corresponding port, it will be sent to the target host B accordingly. As a result, even if a host stole the IP address, but because he did not have this MAC address, and therefore will not receive data packets.

\section{New Data Transfer Model}

The issue of data access has attracted wide attention. HPSS moves very large data objects quickly (several times the speed) between high-performance computers and repositories. It supports parallel file (data partition), parallel data transmission (transmission rate can be up to GBps) and transaction management, the disadvantage is the small file processing efficiency is low, and poor portability. SRB middleware based on the C / S structure provide different types of storage devices consistent access interface. It not only supports archiving file system, operating system file system, but also can support the database, HTTP and FTP, but its MCAT centralized database approach, the introduction of a new access bottleneck. In addition it does not currently support data partition. Globus GridFTP is an important part of the Globus Toolkit. It provides the possibility of striping and parallel transmission, but there is still a lack of collaboration between GridFTP servers. P2P-based systems, such as CAN, focus on file sharing and retrieval issues. Relying on the routing mechanism to locate the resources distributed among the nodes. In addition, they use HTTP as the file transfer protocol. In short, the current research carried out are not well addressed the four issues mentioned above. At present, most systems use two system architectures. One is inheritance Client / Server structure, resources distributed in a small number of high-performance computers, this time requires complex load balancing and fault-tolerant algorithms to provide continuous and reliable access, steadily increase the network bandwidth to handle the request, in addition to the need for cache and Replication mechanism to alleviate the above problems; the other is the use of P2P structures, resources scattered in the various nodes. Such systems avoid many of the C / S architectures, but at the expense of more network bandwidth and increased latency, and introduce more complex node organization, search, security, and copyright issues. The two structures have their own advantages and disadvantages and there is no insurmountable gap between them. When the number of servers increases, the C / S structure changes to the P2P structure; the free-riding phenomenon in the P2P system (70\% of the users do not provide resources, and $50 \%$ of the requests are served by $1 \%$ of the hosts) the actual transformation to the C / S structure. Therefore, GridFTP is designed to be a blend of C / S technology and P2P technology. To achieve four goals, you need three key components: First, the need to design the siteerver is responsible for organizing the region of the data set to form a logical whole. The data set in the site server is represented by the bloom filter. Secondly, the peer network consisting of the Site server is designed to receive, process, or forward the user query request. Finally, it provides high performance data transmission capability.

\section{Implementation of Hybrid Data Transmission Model}

How to design a distributed data collection mechanism with energy efficient, reliable and reliable is a key issue. According to the basic structure of the state monitoring system, it can be seen that the local monitoring network in each vehicle does not need a large number of perceived nodes, and the temporal and spatial correlation of the perceived data is large. The primary task of the first layer sensor network is to monitor the data collection, aggregation and storage, and the amount of data is small. The vehicle monitoring network is relatively independent, and each vehicle can be regarded as a network node. The network topology changes greatly with the relative motion between nodes, 
and there is the vulnerability of network disconnection. The main task of the second layer sensor network is to carry out the aggregation, transmission and storage of vehicle monitoring data and the data volume is large. According to the simulation experiment found that when the number of sensor nodes is small, the use of client / server model data delay is small, time synchronization is good, more efficient; when the sensor nodes are more, the network communication bandwidth requirements More than the network can provide the bandwidth, then use the mobile agent model can avoid a lot of data on the network transmission, better performance.

In this paper, a mixed computing model is used to design the data collection mechanism of the state monitoring system. The client / server calculation model is adopted in the vehicle monitoring network. The calculation model of the mobile agent is adopted between the vehicle monitoring network. In the whole system, the sink node is the hub of connecting two internal and external data collection mechanisms.

In order to reduce RF interference and save energy, the sensor power and communication distance are limited to a small range, the sensor node periodically perceives the relevant information of a geographic area. Whenever a perceptual event is obtained, it is sent to the sink (information receiver, here for the gateway) node. (1) External coding In order to ensure the reliability of monitoring the network, often in the same monitoring object around the deployment of multiple sensor nodes. Therefore, the monitoring area of the different sensor nodes may be partially coincident, and the monitoring data is often relevant. Due to power, occlusion and transmission distance constraints, usually need to use multi-hop communication technology for transmission. In order to save energy, reduce the network transmission congestion and reduce the data transmission delay, when the data flow from the source to the sink node should be aggregated. This technique is often referred to as in-network data aggregation. Before the sensor node forwards data to the sink, the sensor node uses an aggregate function to encode the data. Data decoding is required when the original sensing data needs to be restored at the gateway node.

While aggregating data with outer coding, the source node and the coding node are generally adjacent. So the sensing data mostly occurs at the end of the sensor network. In practice, the temporal and spatial correlation of network nodes is very strong, and the data compression rate is very high. After data aggregation can greatly reduce network traffic, saving communication costs. (2) release / reservation mechanism As the sensor communication power consumption is much larger than the perceived power consumption, data transmission is the most energy-consuming part of the sensor network, in the practical application does not need to let the sensor has been transmitting the sensing data, on-demand query is the most good energy-saving communication mechanism. Some of the communication paradigms currently proposed can be used to handle communication interactions of sensor networks, such as message queues, notifications, shared spaces, and so on. But these paradigms cannot completely remove the coupling between the participants, making the system lack of flexibility and scalability. The publish / reserve paradigm separates the producers and consumers of the information in time, space and traffic. According to the publish / reserve paradigm, sink collects event notifications from the sensor network. When a message of interest is found, the sink issues a reservation to the node that found the event. When the sensor receives a reservation from the network, it activates an event to issue the perceptual data, sending the information to the sink asynchronously. The publish / reserve mechanism can be used to send and receive periodic information based on query and event-driven. In the application, the first need to establish a hop tree to determine the network communication structure. The algorithm of building hop tree is based on flooding mechanism. Starting from the sink, with a hop value, the value is stored, the incremental transmission to the neighbor node. The neighbor node stores the received hop value and increments it to the neighbor node until all the sensor nodes are configured with different hop values. Because the communication between the network nodes through the radio, the node all the neighbors have received the transmission of information. A node that has already been transmitted will receive the transmission of its neighbor, producing a loop. To avoid wasting network energy for invalid transport, use a rule set to assign hop values to nodes. One of the local rules is to compare this value to the local hop value when the node receives a hop value from the 
neighbor. If the local hops value is greater than the received value, the node updates its hop value, increments the value and then re-transmits it to the neighbor node; otherwise it is neither updated nor forwarded. Hop tree establishment process can be assigned to each sensor at the same time the perception task, set the event alarm must meet the threshold, such as temperature $>80 \mathrm{e}$ and so on. After the network is initially configured, the information stored by the node is its hop count, perceived task, and early warning threshold.

\section{Conclusion}

Based on the traditional technology of network data transmission, this paper makes use of parallel transmission and strip transmission technology to improve the transmission performance. By using node self-organization and request routing, the resource location is more relaxed. Using parallel file storage and partial file copy technology, reducing the amount of data redundancy wasted storage space and saving the replication consumed by the network bandwidth.

\section{References}

[1] Han Liqun. Artificial neural network tutorial[M]. Beijing: Beijing University of Posts and Telecommunications Press, 2006

[2] Zhu Daqi, Shi Hui. Artificial neural network principle and application[M]. Beijing: Science Press, 2003

[3] Yang Zhen-shan, Shao Cheng. Preparation of elevator inter-layer traffic distribution based on gray RBF neural network model[J]. Information and Control, 2008, 37 (6): 690 - 696

[4] Liu Lin. Application of fuzzy mathematics [M]. Xi'an: Shaanxi Science and Technology Press, 1996

[5] Mo Hongwei. Artificial immune system principle and application[M]. Harbin: Harbin Institute of Technology Press, 2002

[6] Wei Haikun. Theory and method of neural network structure design [M]. Beijing: National Defense Industry University Press, 2005 